\title{
Limited-Feedback-Based Adaptive Power Allocation and Subcarrier Pairing for OFDM DF Relay Networks With Diversity
}

\author{
Yong Liu and Wen Chen, Senior Member, IEEE
}

\begin{abstract}
A limited-feedback-based dynamic resource allocation algorithm is proposed for a relay cooperative network with orthogonal frequency-division-multiplexing (OFDM) modulation. A communication model where one source node communicates with one destination node assisted by one half-duplex decode-andforward (DF) relay is considered in this paper. We first consider the selective DF scheme, in which some relay subcarriers will keep idle if they do not have the advantage of forwarding the received symbols. Furthermore, we consider the enhanced DF scheme where the idle subcarriers are used to transmit new messages at the source. We aim to maximize the system's instantaneous rate by jointly optimizing power allocation and subcarrier pairing on each subcarrier based on the Lloyd algorithm. Both sum and individual power constraints are considered. The joint optimization turns out to be a mixed integer programming problem. We then transform it into a convex optimization by continuous relaxation and achieve the solution in the dual domain. The performance of the proposed joint resource allocation algorithm is verified by simulations. We find that the proposed scheme outperforms the existing methods in various channel conditions. We also observe that only a few feedback bits can achieve most of the performance gain of the perfect channel-state-information (CSI)-based resource allocation algorithm at different levels of signal-to-noise ratio (SNR).
\end{abstract}

Index Terms-Decode and forward (DF), limited feedback, Lloyd algorithm, orthogonal frequency-division multiplexing (OFDM), power allocation, subcarrier pairing.

\section{INTRODUCTION}

C ONSIDERING the limited budget of transmit power and hardware complexity, cooperative relaying has recently attracted a lot of research interest, which is employed to exploit spatial diversity, combat wireless channel fading, and extend coverage without antenna arrays [1], [2]. For example, IEEE 802.16 currently integrates relays for multihop communications [3]. Two main relay strategies have been adopted in such scenarios: amplify and forward (AF) and decode and forward (DF).

Manuscript received August 23, 2011; revised March 6, 2012 and April 28, 2012; accepted April 29, 2012. Date of publication May 8, 2012; date of current version July 10, 2012. This work was supported by the National 973 Project under Grant 2012CB316106, by the National Science Foundation of China under Grant 60972031 and Grant 61161130529, by the National 973 Project under Grant 2009CB824900, and by the National Key Laboratory Project under Grant ISN11-01. The review of this paper was coordinated by Prof. M. D. Yacoub.

The authors are with the Department of Electronic Engineering, Shanghai Jiao Tong University, Shanghai 200030, China, and also with the State Key Laboratory for Integrated Service Networks, Xidian University, Xi' an 710071, China (e-mail: yongliu1982@sjtu.edu.cn; wenchen@sjtu.edu.cn).

Digital Object Identifier 10.1109/TVT.2012.2198250
The AF relay amplifies and retransmits the received signal without decoding, whereas the latter reencodes the received signal before retransmission.

Orthogonal frequency-division multiplexing (OFDM) is a technique to mitigate frequency selectivity and intersymbol interference with its inherent robustness against frequencyselective fading [4]. Because of its potential for high spectral efficiency, OFDM-based relaying offers a more promising perspective in improving system performance.

Power allocation is always critical in wireless networks due to the limited budget of transmit power. It has been widely discussed in the context of both single-carrier and multicarrier relaying channels [5]-[12]. We have proposed limitedfeedback-based power allocation algorithms for a single-carrier relaying channel and a multicarrier-based relaying model in [5] and [6], respectively. In [7], Ahmed et al. propose a power control algorithm for AF relaying with limited feedback. Then, they study the rate and power control to improve the throughput gain of DF in [8]. On the other hand, power allocation for OFDM-based relaying is also extensively studied. The authors in [9] investigate the power allocation for an OFDM-based AF relaying by separately optimizing the source and relay powers. In [10], the same authors propose a power allocation scheme for multiple-input-multiple-output (MIMO) OFDM relay system in the same way. In [11], Ying et al. work on a similar problem but for DF relaying OFDM systems. Ma et al. introduce power loading algorithms to minimize the transmit power for OFDMbased AF and selective DF modes with respect to various power constraint conditions in [12].

Due to the independent fading on each subcarrier in each hop, subcarrier pairing is employed in OFDM power allocation to further improve system performance [13]-[17]. Most works in the literature focus only on relay models without diversity. A sorted subcarrier pairing scheme is proposed in [13]. The authors determine the pairing sequence by ordering the source-relay (SR) subcarriers and the relay-destination (RD) subcarriers, respectively, according to the channel gains. The authors in [14] prove that the sorted pairing method is optimal for both $\mathrm{DF}$ and $\mathrm{AF}$ relaying without the source-destination (SD) link. In [15], channel pairing, channel user assignment, and power allocation are jointly optimized in a multiple-access system by a polynomial-time algorithm based on continuous relaxation and dual minimization. Wang and $\mathrm{Wu}$ [16] propose a joint subcarrier pairing and power allocation algorithm for an OFDM two-hop relay system with separate power constraints 
and find the solution by separately considering the subcarrier pairing and the power allocation. In [17], optimal subcarrier assignment and power allocation schemes for the multiuser multirelay model is investigated, and the optimal subcarrier and power allocation policy in a quasi-closed form is obtained.

Resource allocation utilizing channel state information (CSI) can yield significant performance improvement [5], [6], [18], [19]. Tremendous innovation that realizes instantaneous channel adaptation is to use feedback whose history may trace back to Shannon [20]. It is proved that with perfect CSI at the source, the error and capacity performance are significantly better than that without CSI [19], [21]. Some research has been carried out to achieve the performance gain based on limited feedback, since perfect CSI at the source is always impractical. One can either send back a quantized CSI or quantized power allocation vectors [7] or the index of the best vector in a power allocation codebook shared by all nodes [22], [23]. These works are mostly studied in point-to-point MIMO and OFDM systems. Only a few works exist on OFDM relay networks. The power allocation issue for a single OFDM AF relay network with limited feedback is investigated in [24]. They construct the codebook based on the Lloyd algorithm. Similarly, Zhang et al. introduce the same idea into the DF model in [25].

In view of the lack of joint optimization of power allocation and subcarrier pairing for OFDM relay systems with diversity based on limited feedback, we aim to solve this problem in this paper. This work is developed based on our previous works [5], [6]. We present a limited-feedback-based joint power allocation and subcarrier pairing for a selective OFDM DF relay network under different levels of quantized CSI feedback. In our feedback scheme, we construct a codebook based on an iterative Lloyd algorithm with a modified distortion measure. The joint optimization problem is formulated as a mixed integer programming problem that is hard to solve. We transform it into a convex problem by continuous relaxation [26], [27] and solve it in the dual-domain instead. In our simulation, we observe that the duality gap virtually turns out to be zero when the number of subcarriers is reasonably large, which is consistent with that observed in [27] and [29].

We then relax the constraint that only the relay can transmit in the relaying phase. When the relay does not transmit on some subcarriers, we employ the enhanced DF that allows the source to transmit new messages on these idle subcarriers. Then, we extend the joint optimization problem for selective DF to that for enhanced DF under both sum power constraint and individual power constraints. It is shown that the extra direct-link transmission leads to a remarkable rate enhancement in the simulation. In addition, some existing schemes such as the conventional uniform power allocation without subcarrier pairing (UPA w/o SP), the optimal power allocation without subcarrier pairing (OPA w/o SP), and the uniform power allocation with subcarrier pairing (UPA with SP) are compared with the proposed algorithm. Simulation results demonstrate that the proposed algorithm outperforms the existing ones. We also find that a negligible performance loss can be achieved with just a few feedback bits at different levels of signal-to-noise ratio (SNR).

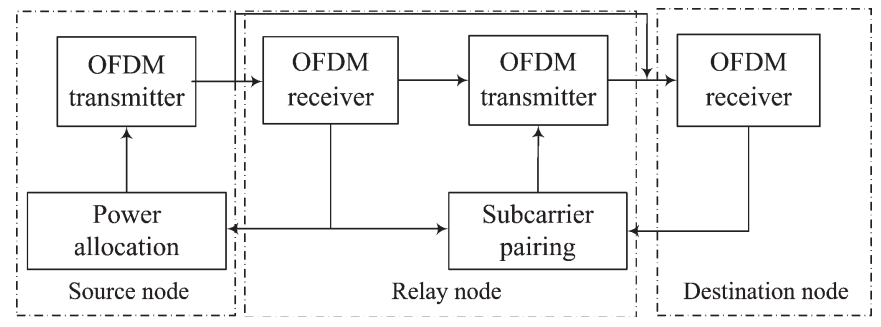

Fig. 1. System block diagram of OFDM cooperative diversity model with limited feedback.

The remainder of this paper is organized as follows. The system model is introduced in Section II. In Section III, we solve the joint optimization problem for selective DF relay networks and propose a limited-feedback-based resource allocation algorithm. In Section IV, we solve the optimization problem for enhanced DF relay networks and then consider the joint optimization under individual power constraints. Simulations are performed in Section V to verify the performance of the proposed algorithm. Finally, the conclusions are drawn in Section VI.

\section{System Model}

The scenario of a three-node DF diversity model is considered, where one source communicates with one destination assisted by one half-duplex relay, as shown in Fig. 1. The channel on each hop is divided into $N$ subcarriers. Communication takes place in two phases. The source broadcasts its signal in the listening phase, whereas the relay and destination listen. The relay decodes and forwards in the relaying phase. It is assumed that each subcarrier in the listening phase is paired with one subcarrier in the relaying phase. Therefore, the number of subcarrier pairs is $N$. We utilize $\operatorname{SP}(m, n)$ to denote the subcarrier $m$ in the listening phase pairing with the subcarrier $n$ in the relaying phase. For subcarrier pair $\operatorname{SP}(m, n)$, it might not be the actual pair participating in communication. If $\operatorname{SP}(m, n)$ actually participates in communication, it is said to be "selected." We denote $h_{\mathrm{SD}}^{m}, h_{\mathrm{SR}}^{m}$, and $h_{\mathrm{RD}}^{n}$ as channel coefficients of the $m$ th subcarrier of SD and SR and the $n$th subcarrier of RD, respectively. For a potential $\mathrm{SP}(m, n)$, the source transmits symbol $s_{m}$ over subcarrier $m$ with power $P_{S}^{m, n}$ in the listening phase, and the received signals at the relay and destination are respectively given by

$$
\begin{aligned}
& y_{r m}=\sqrt{P_{S}^{m, n}} h_{\mathrm{SR}}^{m} s_{m}+z_{r m} \\
& y_{d m}^{(1)}=\sqrt{P_{S}^{m, n}} h_{\mathrm{SD}}^{m} s_{m}+z_{d m}^{(1)}
\end{aligned}
$$

where $z_{d m}^{(1)} \sim \mathcal{C N}\left(0, \sigma_{d}^{2}\right)$ and $z_{r m} \sim \mathcal{C N}\left(0, \sigma_{r}^{2}\right)$ are the additive noises at the relay and the destination, respectively. In the relaying phase, the relay transmits the reencoded signal $\hat{s}_{m}$ with power $P_{R}^{m, n}$ on the $n$th subcarrier, and the received signal at the destination is

$$
y_{d n}^{(2)}=\sqrt{P_{R}^{m, n}} h_{\mathrm{RD}}^{n} \hat{s}_{m}+z_{d n}^{(2)}
$$

where $z_{d n}^{(2)} \sim \mathcal{C N}\left(\mu, \sigma_{d}^{2}\right)$ is the additive noise at the destination in the relaying phase. 
Let $\quad \lambda_{\mathrm{SR}}^{m}=\left|h_{\mathrm{SR}}^{m}\right|^{2} / \sigma_{r}^{2}, \quad \lambda_{\mathrm{RD}}^{n}=\left|h_{\mathrm{RD}}^{n}\right|^{2} / \sigma_{d}^{2}, \quad$ and $\quad \lambda_{\mathrm{SD}}^{m}=$ $\left|h_{\mathrm{SD}}^{m}\right|^{2} / \sigma_{d}^{2}$ denote the normalized channel gains, respectively. Depending on whether the relay is helpful, each subcarrier pair $\mathrm{SP}(m, n)$ may work in either the relaying mode or the idle mode in a selective DF relay [30]. For an $\operatorname{SP}(m, n)$, the relay forwards the message $\hat{s}_{m}$ on subcarrier $n$ in the relaying phase when it works in the relaying mode; while in the idle mode, the relay does not forward $\left(P_{R}^{m, n}=0\right)$, and $s_{m}$ is transmitted to the destination by the SD link in the listening phase only. Then, the end-to-end rate achieved by $\operatorname{SP}(m, n)$ during the two phases is given by (3), shown at the bottom of the page. A criterion to decide the working mode of $\operatorname{SP}(m, n)$, that is, using relay is advantageous when

$$
\min \left\{\lambda_{\mathrm{SR}}^{m}, \lambda_{\mathrm{RD}}^{n}\right\}>\lambda_{\mathrm{SD}}^{m}
$$

in selective DF mode is presented in [11] and [30]. Otherwise, the relay keeps idle on subcarrier $n$ in the relaying phase for $\hat{s}_{m}$.

\section{Limited-FeEdBACK-BASED OptimaL RESOURCE ALLOCATION}

In this section, we analyze the joint optimization of power allocation and subcarrier pairing for selective DF based on limited feedback. The optimization problem is formulated first and then solved in the dual domain.

\section{A. Optimization Problem Formulation}

Let $P^{m, n}=P_{S}^{m, n}+P_{R}^{m, n}$ for $S P(m, n)$. We first consider the rate $R^{m, n}$ in the relaying mode. Then, the sum rate is maximized when

$$
\log _{2}\left(1+P_{S}^{m, n} \lambda_{\mathrm{SR}}^{m}\right)=\log _{2}\left(1+P_{S}^{m, n} \lambda_{\mathrm{SD}}^{m}+P_{R}^{m, n} \lambda_{\mathrm{RD}}^{n}\right)
$$

that is

$$
\left(1+P_{S}^{m, n} \lambda_{\mathrm{SR}}^{m}\right)=\left(1+P_{S}^{m, n} \lambda_{\mathrm{SD}}^{m}+P_{R}^{m, n} \lambda_{\mathrm{RD}}^{n}\right) .
$$

Together with $P^{m, n}=P_{S}^{m, n}+P_{R}^{m, n}$, we obtain

$$
\left\{\begin{array}{l}
P_{S}^{m, n}=\frac{\lambda_{\mathrm{RD}}^{n}}{\lambda_{\mathrm{SR}}^{m}+\lambda_{\mathrm{RD}}^{n}-\lambda_{\mathrm{SD}}^{m}} P^{m, n} \\
P_{R}^{m, n}=\frac{\lambda_{\mathrm{SR}}^{m}-\lambda_{\mathrm{SD}}^{m}}{\lambda_{\mathrm{SR}}^{m}+\lambda_{\mathrm{RD}}^{n}-\lambda_{\mathrm{SD}}^{m}} P^{m, n} .
\end{array}\right.
$$

When the system works in the idle mode, we can easily get

$$
\left\{\begin{array}{l}
P_{S}^{m, n}=P^{m, n} \\
P_{R}^{m, n}=0
\end{array}\right.
$$

Denote $\lambda^{m, n}$ as the equivalent channel gain given by

$$
\lambda^{m, n}= \begin{cases}\frac{\lambda_{\mathrm{SR}}^{m} \lambda_{\mathrm{RD}}^{n}}{\lambda_{\mathrm{SR}}^{m}+\lambda_{\mathrm{RD}}^{n}-\lambda_{\mathrm{SD}}^{m}}, & \text { relaying mode } \\ \lambda_{S, D}^{m}, & \text { idle mode. }\end{cases}
$$

By now, we can unify the rate as

$$
R^{m, n}=\log _{2}\left(1+P^{m, n} \lambda^{m, n}\right) .
$$

We define a subcarrier pairing parameter $t_{m, n} \in\{0,1\}$ that takes 1 if $\operatorname{SP}(m, n)$ is selected, and 0 otherwise. Then, the sum rate optimization problem can be formulated as

$$
\max _{\{\mathbf{P}, \mathbf{t}\}} \sum_{m=1}^{N} \sum_{n=1}^{N} t_{m, n} R^{m, n}
$$

$$
\begin{array}{rr}
\text { s.t. } & \mathbf{C} 1: \sum_{m=1}^{N} \sum_{n=1}^{N} t_{m, n} P^{m, n} \leq P_{t}, \mathbf{C} 2: P^{m, n} \geq 0 \quad \forall m, n \\
\mathbf{C} 3: \sum_{m=1}^{N} t_{m, n}=1 \quad \forall n, \quad \mathbf{C} 4: \sum_{n=1}^{N} t_{m, n}=1 \quad \forall m
\end{array}
$$

where $P_{t}$ is the transmit power budget, and $\mathbf{t}$ and $\mathbf{P}$ are two $N \times N$ matrices with the $(m, n)$ th entry $t_{m, n}$ and $P^{m, n}$, respectively. $\mathbf{C} 3$ and $\mathbf{C} 4$ correspond to the pairing constraint that each subcarrier $m$ in listening phase only pairs with one subcarrier $n$ in the relaying phase.

Since it is a mixed integer programming problem that is difficult to solve, we relax the integer constraint of $t_{m, n} \in$ $\{0,1\}$ as $t_{m, n} \in[0,1] \forall m, n$, as in [26] and [34]. Denote $S^{m, n}=t_{m, n} P^{m, n}$ as the actual power consumed on $\operatorname{SP}(m, n)$. Then, the optimization problem becomes

$$
\begin{aligned}
& \max _{\{\mathbf{S}, \mathbf{t}\}} \sum_{m=1}^{N} \sum_{n=1}^{N} t_{m, n} \frac{1}{2} \log _{2}\left(1+S^{m, n} \frac{\lambda^{m, n}}{t_{m, n}}\right) \\
& \text { s.t. } \quad \text { C5 }: t_{m, n} \geq 0 \quad \forall m, n \\
& \text { C6 }: \sum_{m=1}^{N} \sum_{n=1}^{N} S^{m, n} \leq P_{t} \\
& \text { C7 }: S^{m, n} \geq 0 \quad \forall m, n, \text { and } \mathbf{C} 3-\mathbf{C} 4(12)
\end{aligned}
$$

where $\mathbf{S}=\left(S^{m, n}\right)_{N \times N}$ is an $N \times N$ matrix. Obviously, the preceding objective function is concave with respect to $(\mathbf{S}, \mathbf{t})$. In the following, we will employ the dual method [27], [28] to solve this optimization problem.

In [27], the authors have shown that under a so-called timesharing condition, the duality gap of the optimization problem is always zero, regardless of the convexity of the objective function. Further, the authors show that the time-sharing condition is always satisfied for practical multiuser spectrum optimization problems in multicarrier systems when the number of frequency carriers goes to infinity. This suggests that we can solve the problem by the dual method [28], which will provide an upper bound for the original problem. More importantly, the method can guarantee $t_{m, n}$ being integer valued.

$$
R^{m, n}= \begin{cases}\frac{1}{2} \log _{2}\left(1+P_{S}^{m, n} \lambda_{\mathrm{SD}}^{m}\right), & \text { idle mode } \\ \frac{1}{2} \min \left\{\log _{2}\left(1+P_{S}^{m, n} \lambda_{\mathrm{SD}}^{m}+P_{R}^{m, n} \lambda_{\mathrm{RD}}^{n}\right), \log _{2}\left(1+P_{S}^{m, n} \lambda_{\mathrm{SR}}^{m}\right)\right\}, & \text { relaying mode }\end{cases}
$$




\section{B. Solution by the Dual Method}

Dualizing the constraints $\mathbf{C} 4$ and $\mathbf{C 6}$, we obtain the generated Lagrange function as

$$
\begin{array}{r}
L(\mathbf{S}, \mathbf{t}, \alpha, \boldsymbol{\beta})=\frac{1}{2} \sum_{m=1}^{N} \sum_{n=1}^{N} t_{m, n} \log _{2}\left(1+S^{m, n} \frac{\lambda^{m, n}}{t_{m, n}}\right) \\
+\alpha\left(P_{t}-\sum_{m=1}^{N} \sum_{n=1}^{N} S^{m, n}\right)+\sum_{m=1}^{N} \beta_{m}\left(1-\sum_{n=1}^{N} t_{m, n}\right)
\end{array}
$$

where $\alpha \geq 0$ and $\boldsymbol{\beta}=\left(\beta_{1}, \beta_{2}, \ldots, \beta_{N}\right) \succeq 0$ are dual variables. Then, the dual objective function and the dual problem are, respectively

$$
\begin{aligned}
g(\alpha, \boldsymbol{\beta})= & \max _{\{\mathbf{S}, \mathbf{t}\}} L(\mathbf{s}, \mathbf{t}, \alpha, \boldsymbol{\beta}), \quad \text { s.t. } \mathbf{C} 3, \mathbf{C} 5, \mathbf{C} 6 \\
& \min _{\{\alpha, \boldsymbol{\beta}\}} g(\alpha, \boldsymbol{\beta}), \quad \text { s.t. } \alpha \geq 0, \boldsymbol{\beta} \succeq 0 .
\end{aligned}
$$

Since a dual function is always optimized by first optimizing some variables and then optimizing the remaining ones [28]. We first optimize $S^{m, n}$ with the assumption that $\alpha$ and $\beta_{m}$ are given. Taking the partial differentiation of $L$ with respect to $S^{m, n}$, we have

$$
\frac{\partial L}{\partial S^{m, n}}=\frac{t_{m, n}}{2} \frac{\frac{\lambda_{m, n}}{t_{m, n}}}{1+S^{m, n} \frac{\lambda_{m, n}}{t_{m, n}}}-\alpha=0
$$

that is

$$
\frac{1}{2\left(\frac{S^{m, n}}{t_{m, n}}+\frac{1}{\lambda_{m, n}}\right)}-\alpha=0 .
$$

Together with the constraint $S^{m, n} \geq 0$, we obtain the optimal solution

$$
S_{*}^{m, n}=t_{m, n}\left[\frac{1}{2 \alpha}-\frac{1}{\lambda_{m, n}}\right]^{+}
$$

where $[x]^{+}=\max \{0, x\}$. We find that $S_{*}^{m, n}$ is associated with the subcarrier pairing parameter $t_{m, n}$. To find the optimal solution for $t_{m, n}$, we first substitute (18) into (13) to obtain the updated Lagrange function

$$
\begin{aligned}
L(\mathbf{p}, \mathbf{t}, \alpha, \boldsymbol{\beta})= & \sum_{m=1}^{N} \sum_{n=1}^{N} \frac{t_{m, n}}{2} \log _{2} \\
& \times\left(1+\lambda^{m, n}\left[\frac{1}{2 \alpha}-\frac{1}{\lambda_{m, n}}\right]^{+}\right) \\
& +\alpha\left(P_{t}-\sum_{m=1}^{N} \sum_{n=1}^{N} t_{m, n}\left[\frac{1}{2 \alpha}-\frac{1}{\lambda_{m, n}}\right]^{+}\right) \\
& +\sum_{m=1}^{N} \beta_{m}\left(1-\sum_{n=1}^{N} t_{m, n}\right) \\
= & \sum_{m=1}^{N} \sum_{n=1}^{N} t_{m, n} T_{m, n}+\left(\alpha P_{t}+\sum_{m=1}^{N} \beta_{m}\right)
\end{aligned}
$$

where

$$
\begin{aligned}
T_{m, n}=\frac{1}{2} \log _{2}\left(1+\lambda^{m, n}\right. & {\left.\left[\frac{1}{2 \alpha}-\frac{1}{\lambda_{m, n}}\right]^{+}\right) } \\
& -\alpha\left[\frac{1}{2 \alpha}-\frac{1}{\lambda_{m, n}}\right]^{+}-\beta_{m} .
\end{aligned}
$$

Since both $T_{m, n}$ and $\left(\alpha P_{t}+\sum_{m=1}^{N} \beta_{m}\right)$ are independent of $t_{m, n}$, we obtain the optimal $t_{m, n}^{*}$ for any $n$ as

$$
t_{m, n}^{*}= \begin{cases}1: & m=\arg \max _{m=1, \ldots, N} T_{m, n} \\ 0: & \text { otherwise. }\end{cases}
$$

Suppose that there is a subcarrier $m$ corresponding to two different $n$. Then, it is conflict to the constraint $\mathbf{C} 4$, which is embedded in the Lagrangian. Therefore, $\sum_{m=1}^{N} t_{m, n}^{*}=1 \forall n$. Since both $S_{*}^{m, n}$ and $t_{m, n}^{*}$ include the dual variables $\alpha$ and $\beta_{m}$, we have to find values $\alpha$ and $\beta_{m}$ that minimize $g\left(\alpha, \boldsymbol{\beta}_{m}\right)$. Given $S_{(i)}^{m, n}$ and $t_{m, n}^{(i)}$ in the $i$ th iteration, the optimal values of dual variables can iteratively be achieved by the subgradient method [35]

$$
\left\{\begin{aligned}
\alpha^{(i+1)}= & \alpha^{(i)}-a^{(i)} \\
& \times\left(P_{t}-\sum_{m=1}^{N} \sum_{n=1}^{N} S_{(i)}^{m, n}\right) \\
\beta_{m}^{(i+1)}= & \beta_{m}^{(i)}-b^{(i)}\left(1-\sum_{n=1}^{N} t_{m, n}^{(i)}\right), \quad m=1, \ldots, N
\end{aligned}\right.
$$

in which $i$ is the iteration number, and $a^{(i)}$ and $b^{(i)}$ are step sizes designed properly. Within each iteration, the subcarrier pairing parameter and power allocation vectors can respectively be updated by (18) and (21) with the updated $\alpha$ and $\beta_{m}$. Then, the algorithm to find the optimal resource allocation vectors can be designed as in Algorithm 1.

\section{Algorithm 1 The Optimal Resource Allocation Algorithm}

Step 1: Set $i=1$, and initialize $\alpha^{(i)}, \beta_{m}^{(i)}, \varepsilon$, and $\max _{i t e r}$, Step 2: If $\left(i<\max _{i t e r}\right), a^{(i)}=b^{(i)}=0.01 / \sqrt{i}$,

Step 3: Compute $t_{m, n}^{(i)}$ by (21) using $\alpha=\alpha^{(i)}$ and $\quad \beta_{m}=$ $\beta_{m}^{(i)}$,

Step 4: Compute $S_{(i)}^{m, n}$ by (18) using $\alpha=\alpha^{(i)}$ and $t_{m, n}=$ $t_{m, n}^{(i)}$,

Step 5: Compute $\alpha^{(i+1)}, \beta_{m}^{(i+1)}$ by (22) using $\alpha=\alpha^{(i)}$, $\beta_{m}=\beta_{m}^{(i)}, S^{m, n}=S_{(i)}^{m, n}$, and $t_{m, n}=t_{m, n}^{(i)}$,

Step 6: If $\left(\left|\alpha^{(i+1)}-\alpha^{(i)}\right| /\left|\alpha^{(i+1)}\right|\right)<\varepsilon$ and $\left(\| \beta_{m}^{(i+1)}-\right.$ $\left.\beta_{m}^{(i)}\|/\| \beta_{m}^{(i)} \|\right)<\varepsilon$, exit and output $\alpha^{*}=\alpha^{(i+1)}$, $\boldsymbol{\beta}^{*}=\boldsymbol{\beta}^{(i+1)}, S_{*}^{m, n}=S_{(i)}^{m, n}$, and $t_{m, n}^{*}=t_{m, n}^{(i)}$; otherwise, set $i=i+1$ and go to Step 2 .

Denote the optimal values of the original problem (11), the relaxed problem (12), and the relaxed dual problem (14) as $R_{o}, R_{r}$, and $R_{d}$, respectively. It is obviously $R_{d} \geq R_{r} \geq R_{o}$. 
Because the optimal $t_{m, n}^{*}$ achieved by solving (14) and (15) satisfy $\mathbf{C} 3, \mathbf{C} 4$, and $t_{m, n} \in\{0,1\}, R_{d}$ is also the dual optimum value for problem (11). In our simulation, we find that the duality gap is asymptotically zero when the number of subcarriers is reasonably large. Based on the analysis and simulations of [27] and [29], as well as our paper, it can be concluded that $R_{d} \doteq R_{r} \doteq R_{o}$ for most of the practical cases.

If the subcarrier number is $N$, then the total number of all possible pairing configurations is $N$ !. The complexity of computing the achieved rate (11) is $N$ for a given subcarrier pairing scheme. Thus, the complexity of exhaustive search is $O(N \cdot N !)$, which is prohibitively high. However, within each iteration of Algorithm 1, the complexity of the proposed algorithm is dominated by the computation of (19), which is $O\left(N^{2}\right)$ in terms of logarithm and multiplication operations. The complexity of computing the optimal power allocation and the sum rate is $O(N)$. Therefore, the total complexity for Algorithm 1 is $O\left(k N^{2}\right)$, where $k$ is the number of iterations. It is obvious that the complexity is tractable.

In [27] and [29] it is shown that the duality gap of the optimization problem is always zero when the time sharing condition is satisfied, regardless of the convexity of the objective function. They also showed that the time-sharing condition will be satisfied if the optimal value of the optimization problem is a concave function of the constraints. In our case, the optimal subcarrier pairing may vary as the power constraint changes. Therefore, the maximum sum rate as a function of the sum power constraint may have discrete changes in the slope at the transition points where the optimal subcarrier pairing scheme changes. The sudden jump in the slope might make the optimization nonconcave with the sum power. However, [29] also indicates analytically and through simulations that the concavity will be asymptotically satisfied as the number of subcarriers becomes large. This is because the amount of discrete slope change tends to decrease with more subcarriers since the bandwidth affected by each change becomes narrower. Therefore, the curve is expected to be more concave as the number of subcarriers increases. However, [29] as well as we cannot rigorously prove this in mathematics. In our simulations, we found that the concavity is mostly satisfied when the number of subcarriers is reasonably large, which is consistent with that observed in [29]. For example, when $N=2$, we have observed that only about $0.8 \%$ of the possible channel conditions will result in the nonconcavity, and when $\mathrm{N}=4$, the probability turns to be $0.2 \%$, and the sum rate is almost always concave in the sum power constraint when $N=8$. Therefore, it can be concluded that the duality gap is virtually zero for most of the practical OFDM cases. This will also be verified by our simulation results.

\section{Lloyd Algorithm-Based Codebook Design}

If perfect CSI can be achieved at the source and relay, the resource allocation vectors can be simply determined by Algorithm 1. However, as stated earlier, due to limited resource of feedback link, full knowledge of the CSI available at the transmit sides is difficult in OFDM systems. To solve this problem, we propose a limited feedback algorithm for power allocation and subcarrier pairing in this section. In this algorithm, the destination, which is assumed to have full forward CSI, selects a resource allocation vector from an elaborately designed codebook upon receiving the current CSI and transmits its index to the source and relay through a limited number of feedback bits. This technique employs a codebook of quantized power allocation and subcarrier pairing designed offline and equipped on the source, relay, and destination. The codebook construction for limited-bit feedback can be linked to a vector quantization problem. We use the Lloyd algorithm [23] to search for good resource allocation codebooks based on sum rate criterion.

To design the limited-feedback-based codebook, we have to construct and iteratively use the nearest neighbor rule and the centroid condition, which play crucial roles in the Lloyd algorithm. In the proposed algorithm, the centroid condition is designed to select the optimal codeword with maximum system rate in a given region, whereas the task of the nearest neighbor rule is to determine the region in which the vectors are closest to the optimal codeword of this region. Notice that the optimization of finding the regions and optimal resource allocation scheme is equivalent to designing a vector quantizer with a modified distortion measure [23]. Taking the optimal rate performance as the design criterion, we use the error distance function to measure the average distortion. Using the centroid condition and the nearest neighbor rule iteratively, the error distance will decrease.

Suppose that the destination has perfect CSI $\mathbf{h}=\left(\mathbf{h}_{\mathrm{SD}}, \mathbf{h}_{\mathrm{SR}}\right.$, $\left.\mathbf{h}_{\mathrm{RD}}\right)$, where $\mathbf{h}_{\mathrm{SD}}=\left(h_{\mathrm{SD}}^{1}, \ldots, h_{\mathrm{SD}}^{N}\right), \mathbf{h}_{\mathrm{SR}}=\left(h_{\mathrm{SR}}^{1}, \ldots, h_{\mathrm{SR}}^{N}\right)$, and $\mathbf{h}_{\mathrm{RD}}=\left(h_{\mathrm{RD}}^{1}, \ldots, h_{\mathrm{RD}}^{N}\right)$, respectively, denote the CSIs of $\mathrm{SD}, \mathrm{SR}$, and RD at a particular period. Given $b$ bits of feedback, the space defined by all possible sets of $\mathbf{h}$ is quantized into $\boldsymbol{B}=$ $2^{b}$ regions.

In the sequel, we set codeword as $\mathbf{c}=\left\{\left(P_{S}^{m, n}, P_{R}^{m, n}\right.\right.$, $\left.\left.t_{m, n}\right) \mid m, n=1, \ldots, N\right\}$ and denote $R(\mathbf{c} \mid \mathbf{h})$ as the end-to-end sum rate of a given channel condition $\mathbf{h}$ and codeword $\mathbf{c}$. Then

$$
R(\mathbf{c} \mid \mathbf{h})=\frac{1}{2} \sum_{m=1}^{N} \sum_{n=1}^{N} t_{m, n} \log _{2}\left(1+P^{m, n} \lambda^{m, n}\right) .
$$

We first randomly generate the training channel condition set $\mathbf{H}=\left\{\mathbf{h}_{l}, l=1, \ldots, M\right\}$ with $M \gg B$. Then, we can easily obtain the training code set $\mathbf{T}=\{\mathbf{c}(\mathbf{h}) \mid \mathbf{h} \in \mathbf{H}\}$, in which $\mathbf{c}(\mathbf{h})$ denotes the optimal code achieved by Algorithm 1 for a given $\mathbf{h} \in \mathbf{H}$. The objective of the Lloyd-algorithm-based codebook design is to randomly choose a codebook $\mathbb{C}=\left\{\mathbf{c}_{1}, \mathbf{c}_{2}, \ldots, \mathbf{c}_{B}\right\}$ of size $B$ from the training code set $\mathbf{T}$ and refine it. The error distance function is defined as

$$
D(\mathbb{C})=E_{\mathbf{h} \in \mathbf{H}}\left\{R(\mathbf{c}(\mathbf{h}) \mid \mathbf{h})-\max _{0 \leq k \leq B} R\left(\mathbf{c}_{k} \mid \mathbf{h}\right)\right\}
$$

where $E\{\cdot\}$ is the expectation of a random variable. Using this distortion function, the codebook design algorithm can be summarized as in Algorithm 2. 
Algorithm 2 The Lloyd Algorithm Based Codebook Design

Step 1: Step 1: Set $j=1, \varepsilon>0$, randomly generate the training code set and select the initial codebook

Step 2: $\mathbb{C}_{j}=\left\{\mathbf{c}_{1}^{j}, \mathbf{c}_{2}^{j}, \ldots, \mathbf{c}_{B}^{j}\right\}$ from $\mathbf{T}$, then calculate $D\left(\mathbb{C}_{j}\right)$ by $(24)$;

Step 3: Step 2: Cluster the set of possible channel realization vectors $\mathbf{H}$ into $B$ quantization regions with the $k$ th region

Step 4: $Q_{k}^{j}, k=1, \ldots, B$, denoted as

$Q_{k}^{j}=\left\{\mathbf{h} \mid\left(R\left(\mathbf{c}_{k}^{j} \mid \mathbf{h}\right)\right) \geq\left(R\left(\mathbf{c}_{l}^{j} \mid \mathbf{h}\right)\right) \quad \forall l \in\{1,2, \ldots, B\}\right\}$

Step 5: Step 3: Using (23), generate a new codebook $\mathbb{C}_{j+1}$ with the $k$ th codeword $\mathbf{c}_{k}^{j+1}$ defined as

$$
\mathbf{c}_{k}^{j+1}=\arg \max _{\mathbf{c} \in \mathbf{T}} E_{\mathbf{h} \in Q_{k}^{j}}(R(\mathbf{c} \mid \mathbf{h})), k=1,2, \ldots, B
$$

Step 6: Step 4: Calculate the average distortion $D\left(\mathbb{C}_{j+1}\right)$ by (24);

Step 7: Step 5: If $D\left(\mathbb{C}_{j+1}\right)<D\left(\mathbb{C}_{j}\right)+\varepsilon$ for some small $\varepsilon$, stop iteration and set the optimal $\mathbb{C}^{*}=$ $\mathbb{C}_{j+1}$; otherwise set $j=j+1$ and go back to Step 2 .

While the offline design of codebook seems to be computationally complex and time consuming, the real-time feed back process is quite simple.

\section{Feedback Scheme}

Upon receiving the instantaneous CSI $\mathbf{h}$, the destination searches over all the codewords in the designed codebook of size $B$ and selects the $q$ th codeword provided with maximum sum rate, i.e., $q=\arg \max _{q}\left(R\left(\mathbf{c}_{q} \mid \mathbf{h}\right)\right)$. Afterward, the destination sends back the index $q$ to both the source and the relay through a noiseless feedback link. Since the source and relay have been equipped with the same codebook copies, upon receiving $q$, the source transmits with power $P_{S}^{m, n}$ and the relay with power $P_{R}^{m, n}$ indexed by $q$.

\section{Optimal Resource Allocation FOR ENHANCED DF MODE}

Depending on whether the relay is helpful, each subcarrier pairing may work in either the relaying mode or the idle mode. For a subcarrier pair working in the idle mode, the idle subcarrier in the relaying phase is not utilized. We further allow the source to transmit extra messages on those idle subcarriers in the relaying phase, which is called enhanced DF mode in this paper.

\section{A. Formulation of the Optimization Problem}

Similarly, the achieved rate $R^{m, n}$ of the enhanced DF mode is given at the bottom of the page. $P_{S, 1}^{m, n}, P_{S, 2}^{m, n}$, and $P_{R}^{m, n}$ respectively denote the source power in the listening phase, the source power in the relaying phase, and the relay power in the relaying phase. Because the condition to activate the relay depends not only on the channel gains but also on the power allocation, we define an indicator $\rho_{m, n} \in\{0,1\}$ to show the status of $\operatorname{SP}(m, n)$ at the relay, i.e., the relay is used for $\operatorname{SP}(m, n)$ if $\rho_{m, n}=1$, otherwise, it is not used. Let the equivalent channel gain $\lambda_{1}^{m, n}=\lambda_{\mathrm{SR}}^{m} \lambda_{\mathrm{RD}}^{n} / \lambda_{\mathrm{SR}}^{m}+\lambda_{\mathrm{RD}}^{n}-\lambda_{\mathrm{SD}}^{m}$, and let $P_{\mathrm{SR}}^{m, n}=P_{S, 1}^{m, n}+P_{R}^{m, n}$. Then, the optimization problem based on the sum rate of the enhanced DF mode can be formulated as

$$
\begin{gathered}
\max _{\{\mathbf{P}, \mathbf{t}, \rho\}} \frac{1}{2} \sum_{m=1}^{N} \sum_{n=1}^{N} t_{m, n} \\
\quad \times\left\{\rho_{m, n} \log _{2}\left(1+P_{\mathrm{SR}}^{m, n} \lambda_{1}^{m, n}\right)\right. \\
\quad+\left(1-\rho_{m, n}\right) \log _{2}\left(1+P_{S, 1}^{m, n} \lambda_{\mathrm{SD}}^{m}\right) \\
\left.\quad+\left(1-\rho_{m, n}\right) \log _{2}\left(1+P_{S, 2}^{m, n} \lambda_{\mathrm{SD}}^{n}\right)\right\} \\
\text { s.t. } \mathbf{D} 1: \sum_{m=1}^{N} t_{m, n}=1 \quad \forall n, \sum_{n=1}^{N} t_{m, n}=1 \\
\text { D2 : } \sum_{m=1}^{N} \sum_{n=1}^{N} t_{m, n}\left\{\left(1-\rho_{m, n}\right)\left(P_{S, 1}^{m, n}+P_{S, 2}^{m, n}\right)\right. \\
\text { D3 : } P_{S, 1}^{m, n}, P_{S, 2}^{m, n}, P_{\mathrm{SR}}^{m, n} \geq 0 \\
\left.+\rho_{m, n} P_{\mathrm{SR}}^{m, n}\right\} \leq P_{t}
\end{gathered}
$$

where $\mathbf{p}=\left(P_{\mathrm{SR}}^{m, n}, P_{S, 1}^{m, n}, P_{S, 2}^{m, n}\right) \in\left(\mathbf{R}^{3}\right)^{N \times N}, \mathbf{t}=\left(t_{m, n}\right) \in$ $\mathbf{R}^{N \times N}$, and $\boldsymbol{\rho}=\left(\rho_{m, n}\right) \in \mathbf{R}^{N \times N}$.

Similarly, we make a continuous relaxation to the optimization problem and obtain a standard convex problem. Moreover, we respectively denote $S_{\mathrm{SR}}^{m, n}=t_{m, n} \rho_{m, n} P_{\mathrm{SR}}^{m, n}, S_{S, 1}^{m, n}=$ $t_{m, n}\left(1-\rho_{m, n}\right) P_{S, 1}^{m, n}$, and $S_{S, 2}^{m, n}=t_{m, n}\left(1-\rho_{m, n}\right) P_{S, 2}^{m, n}$ as the actual power consumption at the source and the relay in

$$
R^{m, n}= \begin{cases}\frac{1}{2}\left\{\log _{2}\left(1+P_{S, 1}^{m, n} \lambda_{\mathrm{SD}}^{m}\right)+\log _{2}\left(1+P_{S, 2}^{m, n} \lambda_{\mathrm{SD}}^{n}\right)\right\}, & \text { idle mode } \\ \frac{1}{2} \min \left\{\log _{2}\left(1+P_{S, 1}^{m, n} \lambda_{\mathrm{SR}}^{m}\right), \log _{2}\left(1+P_{S, 1}^{m, n} \lambda_{\mathrm{SD}}^{m}+P_{R}^{m, n} \lambda_{\mathrm{RD}}^{n}\right)\right\}, & \text { relaying mode }\end{cases}
$$


the two phases. Then, the relaxed optimization problem is formulated as

$$
\begin{aligned}
& \max _{\{\mathbf{s , t}, \rho\}} \frac{1}{2} \sum_{m=1}^{N} \sum_{n=1}^{N} t_{m, n} \\
& \times\left\{\rho_{m, n} \log _{2}\left(1+S_{\mathrm{SR}}^{m, n} \frac{\lambda_{1}^{m, n}}{t_{m, n} \rho_{m, n}}\right)\right. \\
&+\left(1-\rho_{m, n}\right) \log _{2}\left(1+S_{S, 1}^{m, n} \frac{\lambda_{\mathrm{SD}}^{m}}{t_{m, n}\left(1-\rho_{m, n}\right)}\right) \\
&\left.\quad+\left(1-\rho_{m, n}\right) \log _{2}\left(1+S_{S, 2}^{m, n} \frac{\lambda_{\mathrm{SD}}^{n}}{t_{m, n}\left(1-\rho_{m, n}\right)}\right)\right\} \\
& \text { s.t. } \quad \text { D4 }: t_{m, n} \geq 0 \quad \forall m n, \mathbf{D} 5: \rho_{m, n} \geq 0 \quad \forall m n \\
& \quad \mathbf{D} 6: \sum_{m=1}^{N} \sum_{n=1}^{N}\left(S_{\mathrm{SR}}^{m, n}+S_{S, 1}^{m, n}+S_{S, 2}^{m, n}\right)=P_{t} \\
& \quad \mathbf{D} 7: S_{S, 1}^{m, n}, S_{S, 2}^{m, n}, S_{\mathrm{SR}}^{m, n} \geq 0 \quad \forall m n, \text { and D1. }
\end{aligned}
$$

\section{B. Dual Solution of the Relaxed Problem}

Dualizing the constraints D1 and D6, we obtain the Lagrangian

$$
\begin{aligned}
& L(\mathbf{s}, \mathbf{t}, \rho, \boldsymbol{\alpha}, \boldsymbol{\beta}) \\
& =\sum_{m=1}^{N} \sum_{n=1}^{N} \frac{t_{m, n}}{2} \\
& \quad \times\left\{\rho_{m, n} \log _{2}\left(1+S_{\mathrm{SR}}^{m, n} \frac{\lambda_{1}^{m, n}}{t_{m, n} \rho_{m, n}}\right)\right. \\
& \quad+\left(1-\rho_{m, n}\right) \log _{2}\left(1+S_{S, 1}^{m, n} \frac{\lambda_{\mathrm{SD}}^{m}}{t_{m, n}\left(1-\rho_{m, n}\right)}\right) \\
& \left.\quad+\left(1-\rho_{m, n}\right) \log _{2}\left(1+S_{S, 2}^{m, n} \frac{\lambda_{\mathrm{SD}}^{n}}{t_{m, n}\left(1-\rho_{m, n}\right)}\right)\right\} \\
& \quad+\alpha\left(P_{t}-\sum_{m=1}^{N} \sum_{n=1}^{N} \sum_{i=1}^{2}\left(S_{S, i}^{m, n}+S_{\mathrm{SR}}^{m, n}\right)\right) \\
& \quad+\sum_{n=1}^{N} \beta_{n}\left(1-\sum_{m=1}^{N} t_{m, n}\right)
\end{aligned}
$$

where $\alpha$ and $\beta_{n}$ are dual variables, as before. Then, the dual objective function and the dual problem can, respectively, be expressed as

$$
\begin{aligned}
g(\alpha, \boldsymbol{\beta})= & \max _{\{\mathbf{s}, \mathbf{t}, \boldsymbol{\rho}\}} L(\mathbf{s}, \mathbf{t}, \boldsymbol{\rho}, \boldsymbol{\alpha}, \boldsymbol{\beta}), \quad \text { s.t. D1, D4-D6 } \\
& \min _{\{\boldsymbol{\alpha}, \boldsymbol{\beta}\}} g(\alpha, \boldsymbol{\beta}), \quad \text { s.t. } \alpha \geq 0, \boldsymbol{\beta} \succeq 0 .
\end{aligned}
$$

Taking derivatives of $L$ with respect to $S_{\mathrm{SR}}^{m, n}, S_{S, 1}^{m, n}$, and $S_{S, 2}^{m, n}$, we obtain the optimal solutions

$$
\begin{aligned}
& S_{S R *}^{m, n}=t_{m, n} \rho_{m, n}\left[\frac{1}{2 \alpha}-\frac{1}{\lambda_{1}^{m, n}}\right]^{+} \\
& S_{S, 1 *}^{m, n}=t_{m, n}\left(1-\rho_{m, n}\right)\left[\frac{1}{2 \alpha}-\frac{1}{\lambda_{\mathrm{SD}}^{m}}\right]^{+} \\
& S_{S, 2 *}^{m, n}=t_{m, n}\left(1-\rho_{m, n}\right)\left[\frac{1}{2 \alpha}-\frac{1}{\lambda_{\mathrm{SD}}^{n}}\right]^{+} .
\end{aligned}
$$

Denote $R_{m, n}^{R}$ as the rate contribution of $\operatorname{SP}(m, n)$ to the Lagrangian in the relaying mode and $R_{m, n}^{I}$ in the idle mode. Then, we have

$$
\begin{aligned}
R_{m, n}^{R}= & \frac{1}{2} \log \left(1+\lambda_{1}^{m, n}\left[\frac{1}{2 \alpha}-\frac{1}{\lambda_{1}^{m, n}}\right]^{+}\right) \\
& -\alpha\left[\frac{1}{2 \alpha}-\frac{1}{\lambda_{1}^{m, n}}\right]^{+} \\
R_{m, n}^{I}= & \frac{1}{2} \log \left(1+\lambda_{\mathrm{SD}}^{m}\left[\frac{1}{2 \alpha}-\frac{1}{\lambda_{\mathrm{SD}}^{m}}\right]^{+}\right) \\
& -\alpha\left[\frac{1}{2 \alpha}-\frac{1}{\lambda_{\mathrm{SD}}^{m}}\right]^{+} \\
& +\frac{1}{2} \log \left(1+\lambda_{\mathrm{SD}}^{n}\left[\frac{1}{2 \alpha}-\frac{1}{\lambda_{\mathrm{SD}}^{n}}\right]^{+}\right) \\
& -\alpha\left[\frac{1}{2 \alpha}-\frac{1}{\lambda_{\mathrm{SD}}^{n}}\right]^{+} .
\end{aligned}
$$

Easily, we obtain the optimal indictor as

$$
\rho_{m, n}^{*}= \begin{cases}1, & \text { when } R_{m, n}^{R}>R_{m, n}^{I} \\ 0, & \text { otherwise. }\end{cases}
$$

Denoting $R_{m, n}^{*}=\rho_{m, n}^{*} R_{m, n}^{R}+\left(1-\rho_{m, n}^{*}\right) R_{m, n}^{I}-\beta_{n}$ for briefness, we obtain the optimal subcarrier pairing parameter as

$$
t_{m, n}^{*}=\left\{\begin{array}{ll}
1, & m=\underset{m=1, \ldots, N}{\arg \max } R_{m, n}^{*} \\
0, & \text { otherwise }
\end{array} \quad \forall n .\right.
$$

We similarly update the Lagrange multipliers $\alpha$ and $\beta$ by subgradient method as

$$
\left\{\begin{aligned}
\alpha^{(i+1)}= & \alpha^{(i)}-a^{(i)} \\
& \times\left\{P_{t}-\sum_{m=1}^{N} \sum_{n=1}^{N}\left(S_{\mathrm{SR}}^{m, n}+S_{S, 1}^{m, n}+S_{S, 2}^{m, n}\right)\right\} \\
\beta_{m}^{(i+1)}= & \beta_{m}^{(i)}-b^{(i)}\left(1-\sum_{n=1}^{N} t_{m, n}^{(i)}\right), \quad m=1, \ldots, N .
\end{aligned}\right.
$$

With the updated $\alpha$ and $\beta_{m}$ in each iteration, we can update the subcarrier pairing $t_{m, n}^{*}$, the power allocation vectors $\left(S_{S R *}^{m, n}, S_{S, 1 *}^{m, n}, S_{S, 2 *}^{m, n}\right)$, and the indicator $\rho_{m, n}^{*}$ by Algorithm 1 . Notice that the iteration procedure in Algorithm 1 should be modified in some places. For example, before computing $t_{m, n}^{(i)}$ in Algorithm 1, we have to figure out $R_{m, n}^{R}, R_{m, n}^{I}$ and $\rho_{m, n}^{*}$ by (54), (34), and (55), respectively. Similarly, we can use Algorithm 2 to design a codebook for limited feedback.

\section{Resource Allocation Under Individual Power Constraints}

In this section, we investigate the resource allocation under individual power constraints for the source and the relay. For the individual power constraints, the sum powers at the source 
and the relay have separate constraints, which can be expressed as

$$
\sum_{m=1}^{N} P_{S}^{m, n} \leq P_{S}, \sum_{n=1}^{N} P_{R}^{m, n} \leq P_{R}
$$

where $P_{S}$ and $P_{R}$ denote the source power constraint and the relay power constraint, respectively. For a given subcarrier pairing $\operatorname{SP}(m, n)$, the mode selection criterion [30], [31] is expressed as

$$
\text { Relaying mode : } \lambda_{\mathrm{SR}}^{m} P_{S}^{m, n} \geq \lambda_{\mathrm{SD}}^{m} P_{S}^{m, n}+\lambda_{\mathrm{RD}}^{n} P_{R}^{m, n} \text {. }
$$

Then, we can similarly obtain a Lagrangian

$$
\begin{aligned}
L= & \sum_{m=1}^{N} \sum_{n=1}^{N} R^{m, n} \\
& +\mu_{S}\left(P_{S}-\sum_{m=1}^{N} P_{S}^{m, n}\right)+\mu_{R}\left(P_{R}-\sum_{n=1}^{N} P_{R}^{m, n}\right) \\
& +\sum_{\{m, n\} \in S_{R}} \rho_{m, n}\left(\lambda_{\mathrm{SR}}^{m} P_{S}^{m, n}-\lambda_{\mathrm{SD}}^{m} P_{S}^{m, n}-\lambda_{\mathrm{RD}}^{n} P_{R}^{m, n}\right)
\end{aligned}
$$

where $S_{R}$ is the SP set of the relaying mode. The Lagrange coefficients $\mu_{S}, \mu_{R} \geq 0$ are chosen such that the individual power constraints are satisfied. The Lagrange multiplier $\rho_{m, n} \geq 0$ corresponds to the mode selection criterion. For almost all of the subcarrier pairs belonging to relaying mode, the authors in [31] conclude that the selection criterion will be satisfied when

$$
\lambda_{\mathrm{SR}}^{m} P_{S}^{m, n}=\lambda_{\mathrm{SD}}^{m} P_{S}^{m, n}+\lambda_{\mathrm{RD}}^{n} P_{R}^{m, n}
$$

with a possible exception pair satisfying $\gamma_{\mathrm{RD}}^{n} / \gamma_{\mathrm{SD}}^{m}=\lambda_{R} / \lambda_{S}$. However, usually, there will be at most one subcarrier pair in this set. Fortunately, we find that the exception $\operatorname{SP}(m, n)$ has the same contribution and cost to the Lagrangian in the model, which are respectively $(1 / 2) \log \left(\lambda_{\mathrm{SD}}^{m} / 2 \mu_{S}\right)$ and $\mu_{S}\left(\left(1 / 2 \mu_{S}\right)-\left(1 / \lambda_{\mathrm{SD}}^{m}\right)\right)$, no matter if it is classified into relaying mode or idle mode. Therefore, we assign it to relaying mode thereafter.

For relaying mode, (41) implies $P_{S}^{m, n}=\left(\lambda_{\mathrm{RD}}^{n} / \lambda_{\mathrm{SR}}^{m}-\right.$ $\left.\lambda_{\mathrm{SD}}^{m}\right) P_{R}^{m, n}$. Then, $P_{S}^{m, n}$ and $P_{R}^{m, n}$ will be zero or positive simultaneously. Thus, in the relaying mode, we can first allocate the total power of $\operatorname{SP}(m, n)$ and then obtain the corresponding $P_{S}^{m, n}$ and $P_{R}^{m, n}$. Let

$$
\left\{\begin{array}{l}
P_{S}^{m, n}=\frac{\lambda_{\mathrm{RD}}^{n}}{\lambda_{\mathrm{SR}}^{m}+\lambda_{\mathrm{RD}}^{n}-\lambda_{\mathrm{SD}}^{m}} P^{m, n} \\
P_{R}^{m, n}=\frac{\lambda_{\mathrm{SR}}^{m}-\lambda_{\mathrm{SD}}^{m}}{\lambda_{\mathrm{SR}}^{m}+\lambda_{\mathrm{RD}}^{n}-\lambda_{\mathrm{SD}}^{m}} P^{m, n}
\end{array}\right.
$$

in the relaying mode, and

$$
\left\{\begin{array}{l}
P_{S}^{m, n}=P^{m, n} \\
P_{R}^{m, n}=0
\end{array}\right.
$$

in the idle mode. Denote the equivalent channel gain of $\mathrm{SP}(m, n)$ by

$$
\bar{\lambda}^{m, n}= \begin{cases}\frac{\lambda_{\mathrm{SR}}^{m} \lambda_{\mathrm{RD}}^{n}}{\lambda_{\mathrm{SR}}^{n}+\lambda_{\mathrm{RD}}^{n}-\lambda_{\mathrm{SD}}^{m}}, & \text { relaying mode } \\ \lambda_{\mathrm{SD}}^{m}, & \text { idle mode. }\end{cases}
$$

We can also use a unified rate expression to demonstrate the original optimization as

$$
R_{m, n}=\frac{1}{2} \log \left(1+\bar{\lambda}^{m, n} P^{m, n}\right) .
$$

The unified rate helps simplifying the optimization in the same way. Let

$$
\begin{aligned}
\mathbf{R}=\sum_{m=1}^{N} & \sum_{n=1}^{N} \frac{t_{m, n}}{2} \\
\times & \quad\left\{\rho_{m, n} \log _{2}\left(1+\frac{P_{1}^{m, n} \bar{\lambda}^{m, n}}{t_{m, n} \rho_{m, n}}\right)+\left(1-\rho_{m, n}\right)\right. \\
\quad & \quad\left[\log _{2}\left(1+\frac{P_{2}^{m, n} \lambda_{\mathrm{SD}}^{m}}{t_{m, n}\left(1-\rho_{m, n}\right)}\right)\right. \\
& \left.\left.\quad+\log _{2}\left(1+\frac{P_{3}^{m, n} \lambda_{\mathrm{SD}}^{n}}{t_{m, n}\left(1-\rho_{m, n}\right)}\right)\right]\right\}
\end{aligned}
$$

where $P_{1}^{m, n}$ is the sum power of $\operatorname{SP}(m, n)$ in the relaying mode, which can be obtained from (42). $P_{2}^{m, n}$ and $P_{3}^{m, n}$ are respectively the powers used by the direct link of $\operatorname{SP}(m, n)$ in the listening and relaying phases. Then, the sum rate optimization is formulated as

$$
\begin{array}{ll} 
& \max _{\{\mathbf{S}, \mathbf{t}, \boldsymbol{\rho}\}} \mathbf{R}, \\
\text { s.t. } & \mathbf{E} 1: \sum_{m=1}^{N} t_{m, n}=1 \quad \forall n, \mathbf{E} 2: \sum_{n=1}^{N} t_{m, n}=1 \quad \forall m \\
& \mathbf{E} 3: \rho_{m, n} \in\{0,1\}, \quad \mathbf{E} 4: t_{m, n} \in\{0,1\} \\
& \text { E5 : } \sum_{m=1}^{N} \sum_{n=1}^{N}\left(\eta_{S}^{m, n} P_{1}^{m, n}+P_{2}^{m, n}+P_{3}^{m, n}\right) \leq P_{S} \\
& \text { E6 : } \sum_{m=1}^{N} \sum_{n=1}^{N} \eta_{R}^{m, n} P_{1}^{m, n} \leq P_{R}, \mathbf{E} 7: P_{j}^{m, n} \geq 0 \quad \forall j .
\end{array}
$$

Let $\mathbf{P}=\left(P_{1}^{m, n}, P_{2}^{m, n}, P_{3}^{m, n}\right) \in \mathbb{R}^{N \times N \times 3}, \mathbf{t}=\left(t_{m, n}\right) \in \mathbb{R}^{N \times N}$ and $\boldsymbol{\rho}=\left(\rho_{m, n}\right) \in \mathbb{R}^{N \times N}$. Denote

$$
\begin{aligned}
\eta_{S}^{m, n} & = \begin{cases}\frac{\lambda_{R, D}^{n}}{\lambda_{\mathrm{SR}}^{m}+\lambda_{\mathrm{RD}}^{n}-\lambda_{\mathrm{SD}}^{m}}, & \text { relaying mode } \\
1, & \text { idle mode }\end{cases} \\
\eta_{R}^{m, n} & = \begin{cases}\frac{\lambda_{\mathrm{SR}}^{m}-\lambda_{\mathrm{SD}}^{m}}{\lambda_{\mathrm{SR}}^{m}+\lambda_{\mathrm{RD}}^{n}-\lambda_{\mathrm{SD}}^{m}}, & \text { relaying mode } \\
0, & \text { idle mode. }\end{cases}
\end{aligned}
$$

We dualize the constraints E1, E5, E6, and (41). Then, the generated Lagrange function is

$$
\begin{aligned}
& L\left(\mathbf{P}, \mathbf{t}, \lambda_{S}, \lambda_{R}, \boldsymbol{\beta}\right) \\
& \quad=\mathbf{R}+\sum_{n=1}^{N} \beta_{n}\left(1-\sum_{m=1}^{N} t_{m, n}\right)
\end{aligned}
$$




$$
\begin{aligned}
& +\mu_{S}\left(P_{S}-\sum_{m=1}^{N} \sum_{n=1}^{N}\left(\eta_{S}^{m, n} P_{1}^{m, n}-P_{2}^{m, n}-P_{3}^{m, n}\right)\right) \\
& +\mu_{R}\left(P_{R}-\sum_{m=1}^{N} \sum_{n=1}^{N} \eta_{R}^{m, n} P_{1}^{m, n}\right)
\end{aligned}
$$

where $\mu_{S} \geq 0, \mu_{R} \geq 0$, and $\boldsymbol{\beta}=\left(\beta_{1}, \beta_{2}, \ldots, \beta_{N}\right) \succeq 0$ are dual variables. The dual objective function is

$$
\begin{aligned}
g\left(\mu_{S}, \mu_{R}, \boldsymbol{\beta}\right) & =\max _{\{\mathbf{P}, \mathbf{t}, \boldsymbol{\rho}\}} L\left(\mathbf{P}, \mathbf{t}, \mu_{S}, \mu_{R}, \boldsymbol{\beta}, \boldsymbol{\rho}\right) \\
\text { s.t. } & \mathbf{E} 2, \mathbf{E} 7, \mathbf{E} 8, \mathbf{E} 9
\end{aligned}
$$

and the dual problem is

$$
\min _{\left\{\mu_{S}, \mu_{R}, \boldsymbol{\beta}\right\}} g\left(\mu_{S}, \mu_{R}, \boldsymbol{\beta}\right), \quad \text { s.t. } \quad \mu_{S} \geq 0, \mu_{R} \geq 0 .
$$

We take derivatives of $L$ with respect to $P_{1}^{m, n}, P_{2}^{m, n}$, and $P_{3}^{m, n}$ and obtain

$$
\begin{aligned}
& P_{1 *}^{m, n}=t_{m, n} \rho_{m, n}\left[\frac{1}{2\left(\mu_{S} \eta_{S}^{m, n}+\mu_{R} \eta_{R}^{m, n}\right)}-\frac{1}{\bar{\lambda}^{m, n}}\right]^{+} \\
& P_{2 *}^{m, n}=t_{m, n}\left(1-\rho_{m, n}\right)\left[\frac{1}{2 \mu_{S}}-\frac{1}{\lambda_{\mathrm{SD}}^{m}}\right]^{+} \\
& P_{3 *}^{m, n}=t_{m, n}\left(1-\rho_{m, n}\right)\left[\frac{1}{2 \mu_{R}}-\frac{1}{\lambda_{\mathrm{SD}}^{n}}\right]^{+} .
\end{aligned}
$$

Denote $R_{m, n}^{R}$ and $R_{m, n}^{I}$ as the rate contributions of $\operatorname{SP}(m, n)$ to the Lagrange function in relaying and idle modes, respectively. Then

$$
\begin{aligned}
R_{m, n}^{R}= & \frac{1}{2} \log \left(1+\bar{\lambda}^{m, n} \tilde{P}_{1 *}^{m, n}\right)-\mu_{S} \eta_{S}^{m, n} \tilde{P}_{1 *}^{m, n} \\
& -\mu_{R} \eta_{R}^{m, n} \tilde{P}_{1 *}^{m, n} \\
R_{m, n}^{I}= & -\mu_{S}\left(\tilde{P}_{2 *}^{m, n}+\tilde{P}_{3 *}^{m, n}\right) \\
& +\frac{1}{2}\left[\log \left(1+\lambda_{\mathrm{SD}}^{m} \tilde{P}_{2 *}^{m, n}\right)+\log \left(1+\lambda_{\mathrm{SD}}^{n} \tilde{P}_{3 *}^{m, n}\right)\right]
\end{aligned}
$$

where $\tilde{P}_{1 *}^{m, n}=\left[\left(1 / 2\left(\mu_{S} \eta_{S}^{m, n}+\mu_{R} \eta_{R}^{m, n}\right)\right)-\left(1 / \bar{\lambda}^{m, n}\right)\right]^{+}, \tilde{P}_{2 *}^{m, n}=$ $\left[\left(1 / 2 \mu_{S}\right)-\left(1 / \lambda_{\mathrm{SD}}^{m}\right)\right]^{+}$, and $\tilde{P}_{3 *}^{m, n}=\left[\left(1 / 2 \mu_{R}\right)-\left(1 / \lambda_{\mathrm{SD}}^{n}\right)\right]^{+}$. We easily obtain

$$
\rho_{m, n}^{*}= \begin{cases}1, & \text { when } R_{m, n}^{R}>R_{m, n}^{I} \\ 0, & \text { otherwise }\end{cases}
$$

Substituting (53) into (50), we obtain

$$
L\left(\mathbf{P}, \mathbf{t}, \mu_{S}, \mu_{R}, \boldsymbol{\beta}, \boldsymbol{\rho}\right)=t_{m, n} T_{m, n}+K_{\mu_{S}, \mu_{R}, \boldsymbol{\beta}}
$$

where $\quad T_{m, n}=\rho_{m, n} R_{m, n}^{R}+\left(1-\rho_{m, n}\right) R_{m, n}^{N R}-\beta_{n}, \quad$ and $K_{\mu_{S}, \mu_{R}, \boldsymbol{\beta}}=\mu_{S} P_{S}+\mu_{R} P_{R}+\sum_{n=1}^{N} \beta_{n}$. Both $T_{m, n}$ and $K_{\mu_{S}, \mu_{R}, \boldsymbol{\beta}}$ are independent of $t_{m, n}$. Therefore, the optimal $t_{m, n}^{*}$ is obtained as

$$
t_{m, n}^{*}=\left\{\begin{array}{ll}
1: & m=\arg \max _{m=1, \ldots, N} T_{m, n} \\
0: & n \text { otherwise }
\end{array} \quad \forall n .\right.
$$

$\mu_{S}, \mu_{R}$, and $\beta_{n}$ that minimize $g\left(\mu_{S}, \mu_{R}, \boldsymbol{\beta}\right)$ are achieved by the subgradient method

$$
\left\{\begin{aligned}
\mu_{S}^{(i+1)}=\mu_{S}^{(i)}-a^{(i)} & \left(P_{S}-\sum_{m=1}^{N} \sum_{n=1}^{N}\right. \\
& \left.\quad \times\left(\eta_{S}^{m, n} P_{1(i)}^{m, n}-P_{2(i)}^{m, n}-P_{3(i)}^{m, n}\right)\right) \\
\mu_{R}^{(i+1)}=\mu_{R}^{(i)}-b^{(i)} & \left(P_{R}-\sum_{m=1}^{N} \sum_{n=1}^{N} \eta_{R}^{m, n} P_{1(i)}^{m, n}\right) \\
\beta_{n}^{(i+1)}=\beta_{n}^{(i)}-c^{(i)} & \left(1-\sum_{m=1}^{N} t_{m, n}^{(i)}\right), n=1, \ldots, N .
\end{aligned}\right.
$$

By now, we have obtained the optimal mode selection vector, subcarrier pairing vector $t_{m, n}^{*}$, and power allocation vector $\left(P_{1 *}^{m, n}, P_{2 *}^{m, n}, P_{3 *}^{m, n}\right)$ for the given dual variables, respectively. We can similarly update the subcarrier pairing and power allocation vectors as in Algorithm 1 with some slight modifications. The Lloyd algorithm can be employed again to design the codebook.

\section{Simulation Results}

We present some simulations to demonstrate the performance of the proposed algorithms in this section. The channels of the subcarriers are independent identically distributed (i.i.d.) subject to Rayleigh fading, with a large-scale fading path loss exponent 2.5. The channel coefficients are assumed to be constant within two phases and varying independently from one period to another. We assume equal noise power at relay and destination nodes, i.e., $\sigma_{r}^{2}=\sigma_{d}^{2}$. In the simulations, quadrature phase-shift keying modulation is adopted, and the step sizes $a^{(i)}$ and $b^{(i)}$ for the subgradient method are set to be $(0.01 / \sqrt{i})$, where $i$ is the iteration index. The size of the CSI set in Algorithm 2 is $10^{4}$, which is far more than the quantized regions. Several existing schemes are compared with the proposed algorithm in terms of sum rate. These existing schemes include the following:

i) UPA w/o SP: The messages transmitted on subcarrier $m$ at the source node will be retransmitted on subcarrier $m$ at the relay node; the power is allocated equally at the source and relay subcarriers.

ii) OPA w/o SP: The messages transmitted on the subcarrier $m$ at the source node will be retransmitted on the subcarrier $m$ at the relay node; the power allocation is performed according to waterfilling at the source and the relay subcarriers.

iii) UPA with SP: The messages transmitted on the subcarrier $m$ at the source node will be retransmitted on subcarrier $n$ at the relay node by subcarrier pairing; the power is allocated equally at the source and relay subcarriers.

Then, the performance of the proposed algorithm with different feedback bits is demonstrated. In addition, the performance gap between the enhanced DF and the selective DF modes versus the subcarrier number is also revealed in our simulations.

\section{A. Rate Comparison for Different Schemes}

Schemes 1 and 3 are compared with the proposed algorithm with perfect CSI and limited feedback scheme in Fig. 2. The upper curve denotes the proposed joint power allocation and 


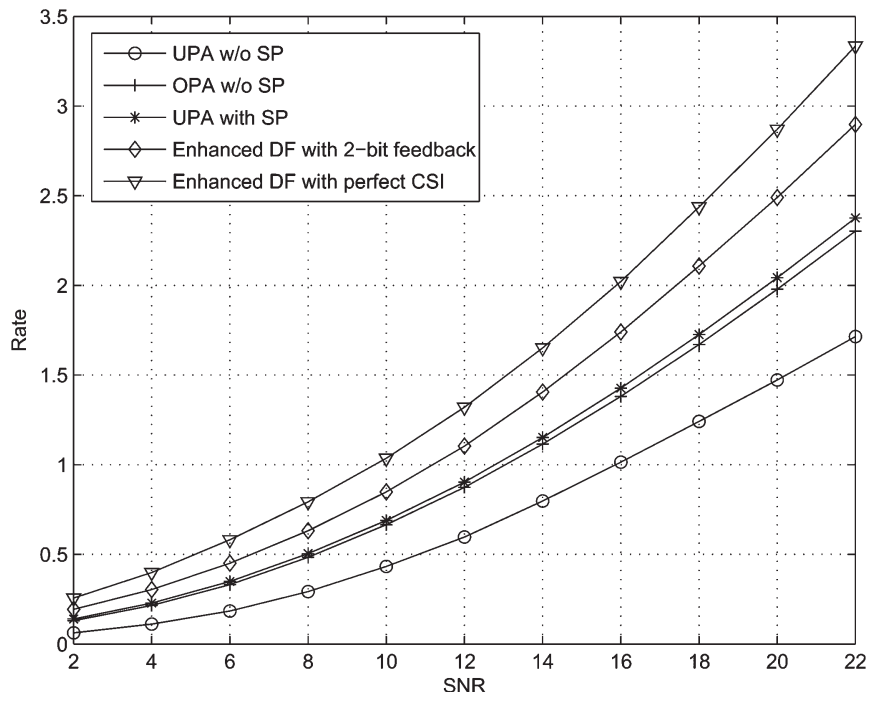

Fig. 2. System sum rate versus SNR for the proposed enhanced DF relay schemes and the existing schemes, where "w/o" denotes "without."

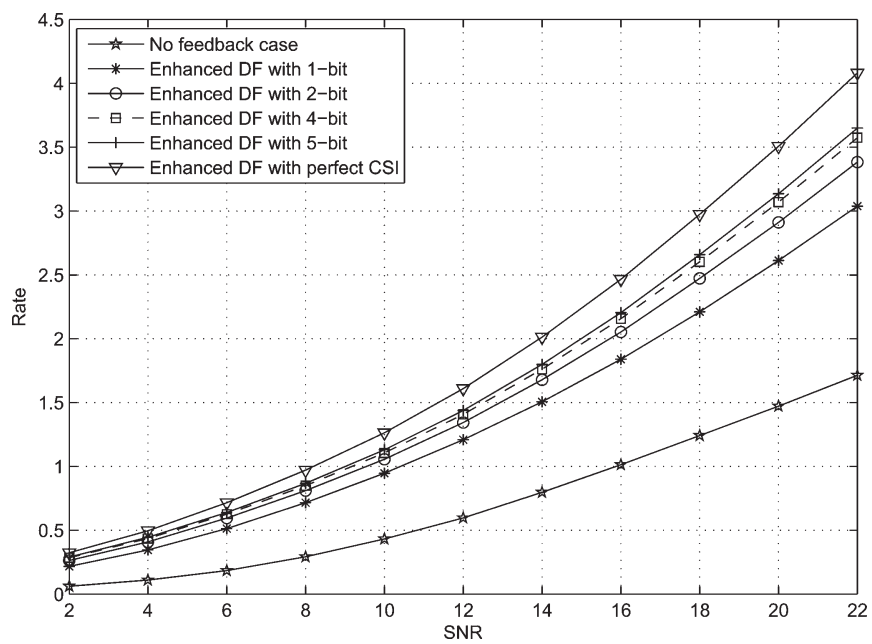

Fig. 3. System sum rate versus SNR for the proposed enhanced DF relay scheme with different levels of feedback bits. The upper curve denotes the perfect CSI case, and the lowest curve denotes the scheme without feedback, where the power is uniformly allocated. Other curves demonstrate the effect of different feedback bits on sum rate.

subcarrier pairing for the enhanced DF scheme with perfect CSI. The second upper curve denotes the proposed joint power allocation and subcarrier pairing for the enhanced DF scheme with 2-bit feedback. The other three curves denote the existing schemes 1 and 3, respectively. We can observe that, only with 2-bit feedback, the proposed joint power allocation and subcarrier pairing for the enhanced DF relay outperforms the existing schemes 1 and 3 greatly. Therefore, we can conclude that the joint power allocation and subcarrier pairing make valuable contribution to system sum rate.

\section{B. Rate Comparison for Different Feedback Bits}

The joint power allocation and subcarrier pairing for the enhanced DF relay with different feedback bits are compared in Fig. 3. We can find that only a few feedback bits are enough to

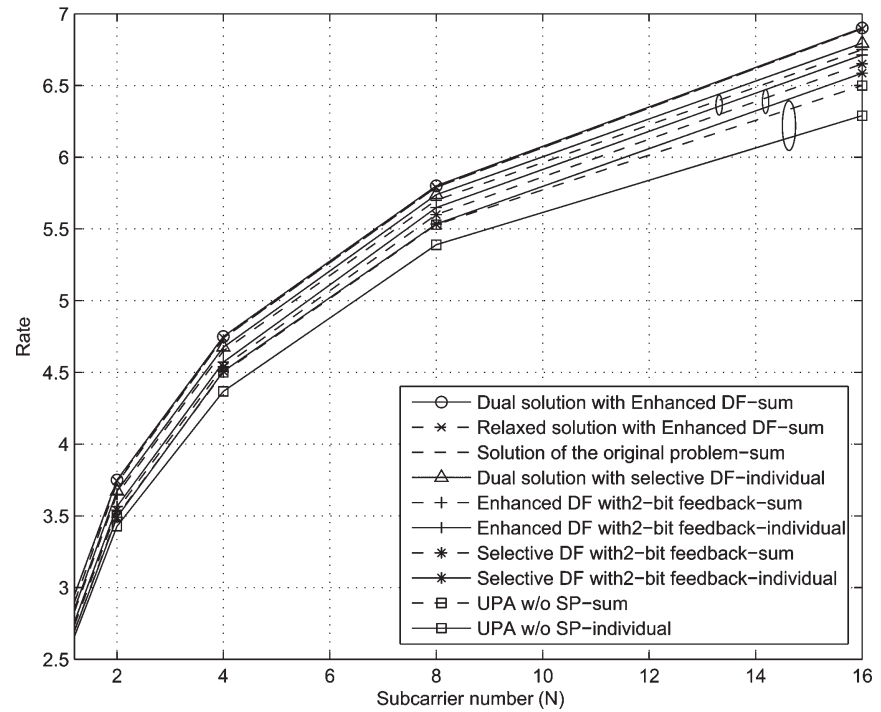

Fig. 4. Sum rate versus the number of subcarriers for the enhanced DF and selective DF with fixed feedback bit of 2 . These curves are obtained with $d=0.4$.

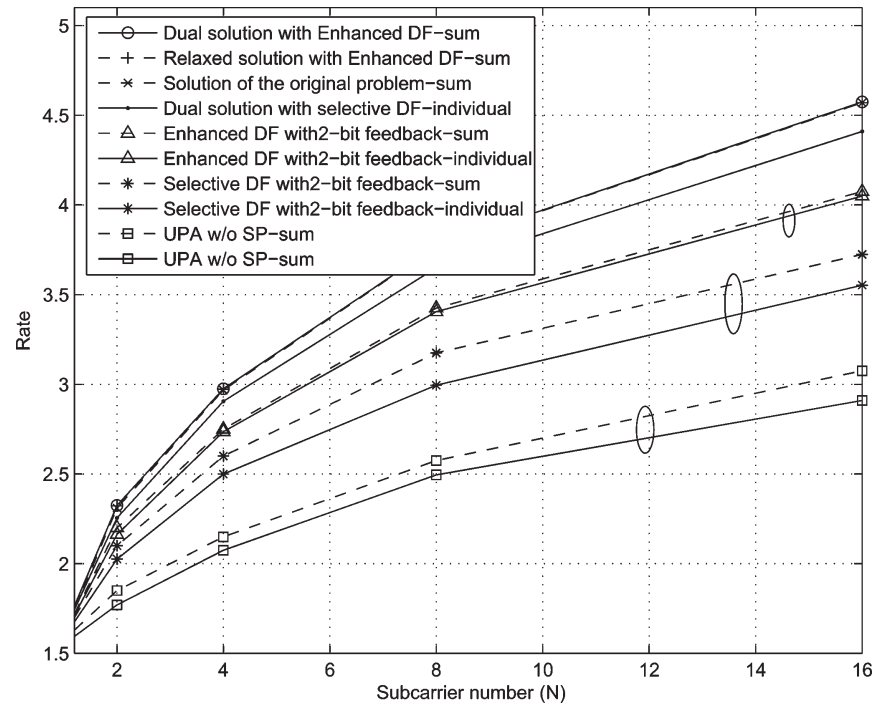

Fig. 5. Sum rate versus the number of subcarriers and the performance gap between the schemes with modified idle and selective relaying modes. We assume that system operates with fixed feedback bit level of 2 . The curves are obtained with $d=0.8$

achieve most of the performance gains of the perfect feedback. For example, with 4 bits of feedback at a rate of 2.5 in Fig. 3, there is only a $-1.7-\mathrm{dB}$ gap to the perfect CSI case, and we also notice that further increasing the feedback bits brings degressive improvement, which implies that the feedback bits as well as the codebook size in the model are not necessarily too large.

\section{Rate Versus Different Subcarrier Numbers Under Sum and Individual Power Constraints}

With the sum and individual power constraints, the sum rates versus the number of subcarriers for the selective DF and the enhanced DF relaying modes are illustrated in Figs. 4 and 5. We 


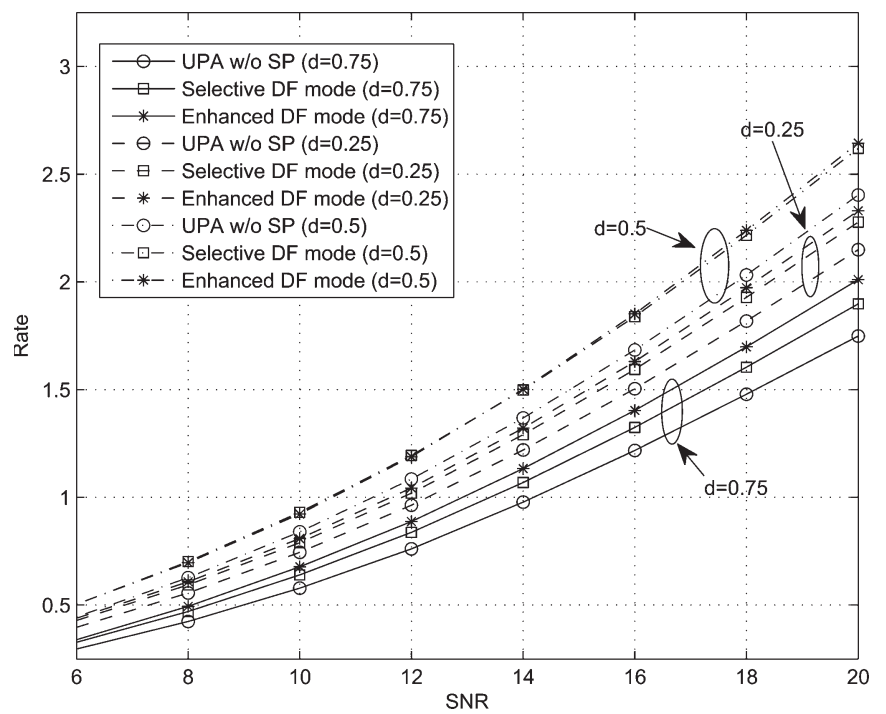

Fig. 6. Sum rate versus average SNR for $d=0.25,0.5,0.75$, with feedback bits of 2 .

consider the cases that subcarrier number $N=2,4,8,16$ with fixed feedback bit level of 2 . For the sake of fairness, we assume $P_{S}+P_{R}=P_{t}$. In addition, as for the case with individual power constraints, we assume $P_{S}=3 P_{R}$. The constraints are set with the practical consideration that the relay often plays the role of assisting the transmission between the source and the destination. Moreover, if more power is assigned to the relay node, the achievable rate will be limited since some of the relay power will not be used. Assuming $P_{S}=(3 / 4) P_{t}$ will make the comparison with the sum power constraints much fairer. In addition, we assume that the relay locates in a line between the source and the destination, and the SD distance is one unit. Denote $d(0<d<1)$ as the SR distance. Thus, the $\mathrm{RD}$ distance is $1-d$. Fig. 4 is obtained with $d=0.4$, whereas $d=0.8$ in Fig. 5. We find that the enhanced DF mode always outperforms the selective DF mode and the schemes without subcarrier pairing, especially when the channel condition of $\mathrm{RD}$ is relatively poor. We can also observe that the bigger the subcarrier number is, the bigger the performance gap between the two modes is. As for the cases under different constraints, the performance of the sum power constraint is better than that of the individual power constraints, which is due to the more flexibility of power allocation between source and relay under the sum power constraint. In addition, we consider the duality gaps in the two figures. The simulation results exactly coincide with our analysis in Section III. We find that the dual solutions approximate to the optimal values of (47) in our simulation. The duality gaps turn out to be nearly 0 when the number of subcarriers is reasonably large, which is consistent with the prediction in [29].

\section{Effect of Relay Location On Rate}

To exploit the system rate versus SNR for different relay locations, we simulate the rate versus SNR by setting $d=0.25$, $d=0.5$, and $d=0.75$, respectively. Fig. 6 demonstrates the effect of relay location to system sum rate at different SNRs

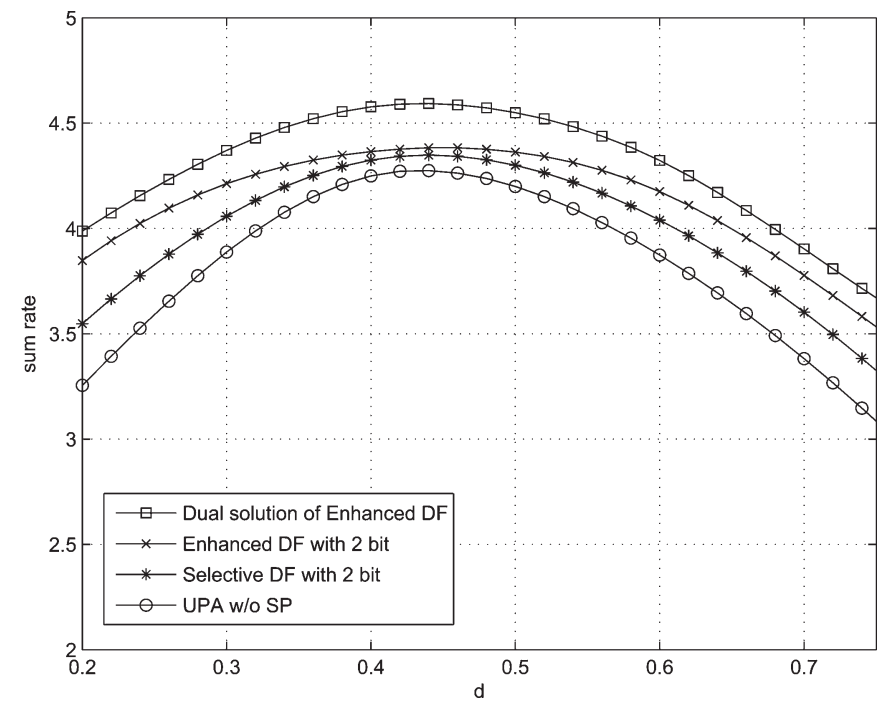

Fig. 7. Sum rate versus the relay location for the enhanced DF and selective DF with 2-bit feedback when $N=4$.

with a fixed feedback bit level of 2 . We can find that the enhanced DF mode always outperforms the selective DF mode and the OPA w/o SP in any kind of $d$, and the channel condition of SR plays a more important role than the channel condition of RD in general. In addition, we find that the gap between the system sum rates achieved by the enhanced DF and the selective DF is larger when $|d-0.5|$ is larger, whereas the performance gap between the enhanced DF and the selective DF is tiny when $d=0.5$.

To exploit the effect of relay location to the system performance, we simulate the sum rate versus relay location $d$ in Fig. 7. The figure is obtained with the fixed feedback bit 2 and subcarrier number $N=4$. We find that the rate reaches maximum at about $d=0.45$. We also observe that comparing with the proposed scheme with 2-bit feedback, the performance loss of the scheme UPA with SP is not very big at this location, which implies that if none of SR or RD channels is very poor, or there is no great difference between the channel conditions of SR and RD, and the scheme UPA with SP can provide acceptable performance with $N=4$. However, if at least one of these channel conditions is very poor, we would be better to dynamically allocate the power and subcarrier resources, since the proposed algorithm can achieve remarkable performance gain. In addition, the performance loss of the scheme UPA with SP increases with the number of subcarriers due to frequency diversity and more flexibility in pairing of large $N$. Figs. 8 and 9 are obtained with $N=32$ and 64, respectively. We observe that the performance gains of the proposed algorithm are much more remarkable. The remarkable performance gain results from the much more pairing degree provided by the big subcarrier number. There is another general trend that can be observed from the two figures. The rate gap between the enhanced DF and the selective DF is larger when $|d-0.5|$ is larger. The performance improvement of the enhanced DF is due to the extra direct-link transmission in the second phase, since the relay has a high possibility of being idle when the $S-R$ or $R-D$ channel is poor because of the large $|d-0.5|$. 


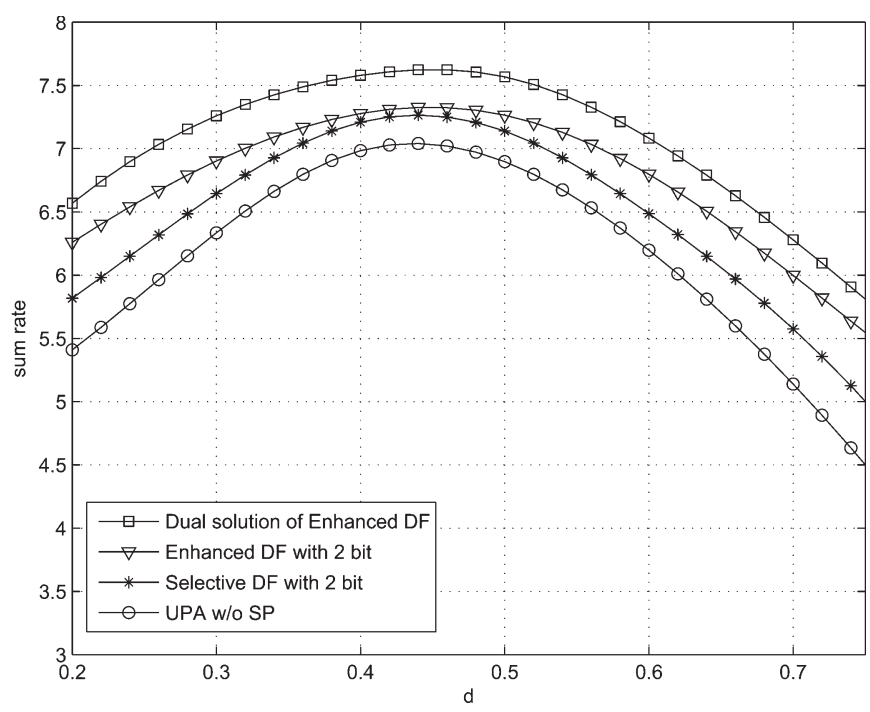

Fig. 8. Sum rate versus the relay location for the enhanced DF and selective DF with 2-bit feedback when $N=32$.

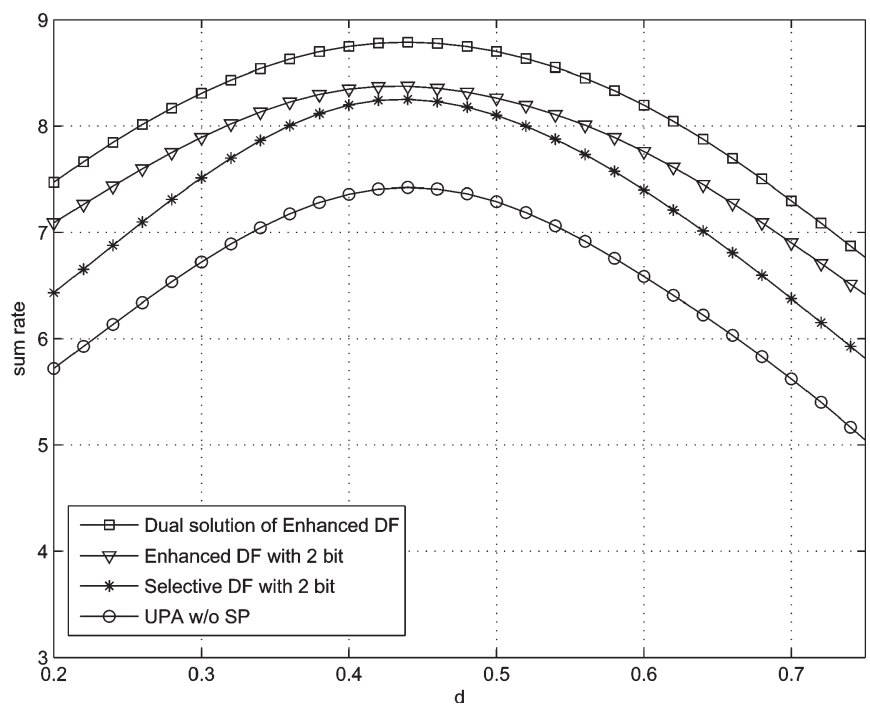

Fig. 9. Sum rate versus the relay location for the enhanced DF and selective DF with 2-bit feedback when $N=64$.

\section{CONCLUSION}

In this paper, we have discussed a limited-feedback-based joint power allocation and subcarrier pairing algorithm for OFDM DF relay networks with diversity. When the relay does not forward the received symbols on some subcarriers, we further allow the source node to transmit new messages on these idle subcarriers. Both sum power constraint and individual power constraints for the source and relay nodes are considered. Since the formulated optimization is a mixed integer programming problem, we transform it into a convex problem by continuous relaxation and then solve it in the dual domain. Simulations show that the proposed algorithms can achieve considerable rate gain with tractable complexities. It outperforms several existing schemes under various channel conditions. The contribution of the extra direct-link transmission is also clearly demonstrated in the simulation. In addition, we notice that a negligible performance loss can be achieved with just a few feedback bits at different levels of SNR values.

\section{REFERENCES}

[1] J. N. Laneman, D. N. C. Tse, and G. W. Wornell, "Cooperative diversity in wireless networks: Efficient protocols and outage behavior," IEEE Trans. Inf. Theory, vol. 50, no. 12, pp. 3062-3080, Dec. 2004.

[2] A. Host Madsen and J. Zhang, "Capacity bounds and power allocation in wireless relay channel," IEEE Trans. Inf. Theory, vol. 51, no. 6, pp. 2020-2040, Jun. 2005.

[3] C. Hoymann, K. Klagges, and M. Schinnenberg, "Multihop communication in relay enhanced IEEE 802.16 networks," in Proc. IEEE PIMRC, Sep. 2006, pp. 1-4.

[4] N. R. Van and P. Ramjee, OFDM for Wireless Multimedia Communications. Boston, MA: Artech House, 2000.

[5] Y. Liu and W. Chen, "Power allocation for the fading relay channel with limited feedback," in Proc. IEEE ICC, May 2010, pp. 1-5.

[6] Y. Liu, W. Chen, and X. P. Huang, "Capacity based adaptive power allocation for the OFDM relay networks with limited feedback," in Proc. IEEE ICC, Jun. 2011, pp. 1-5.

[7] N. Ahmed, M. A. Khojastepour, A. Sabharwal, and B. Aazhang, "Outage minimization with limited feedback for the fading relay channels," IEEE Trans. Commun., vol. 54, no. 4, pp. 659-669, Apr. 2006.

[8] N. Ahmed and B. Aazhang, "Throughput gains using rate and power control in cooperative relay networks," IEEE Trans. Commun., vol. 55, no. 4, pp. 656-660, Apr. 2007.

[9] I. Hammerstrom and A. Wittneben, "On the optimal power allocation for nonregenerative OFDM relay links," in Proc. IEEE ICC, Jun. 2006, vol. 10, pp. 4463-4468.

[10] I. Hammerstrom and A. Wittneben, "Power allocation schemes for amplify and forward MIMO-OFDM relay links," IEEE Trans. Wireless Commun., vol. 6, no. 8, pp. 2798-2802, Aug. 2007.

[11] W. Ying, Q. Xin-chun, W. Tong, and L. Bao-ling, "Power allocation and subcarrier pairing algorithm for regenerative OFDM relay system," in Proc. IEEE VTC, Apr. 2007, pp. 2727-2731.

[12] Y. Ma, N. Yi, and R. Tafazolli, "Bit and power loading for OFDM based three-node relaying communications," IEEE Trans. Signal Process., vol. 56, no. 7, pp. 3236-3247, Jul. 2008.

[13] M. Herdin, "A chunk based OFDM amplify-and-forward relaying scheme for 4G mobile radio systems," in Proc. IEEE ICC, Istanbul, Turkey, Jun. 2006, vol. 10, pp. 4507-4512.

[14] Y. Li, W. Wang, J. Kong, and M. Peng, "Subcarrier pairing for amplify-and-forward and decode-and-forward OFDM relay links," IEEE Commun. Lett., vol. 13, no. 4, pp. 209-211, Apr. 2009.

[15] M. Hajiaghayi, M. Dong, and B. Liang, "Optimal channel assignment and power allocation for dual-hop multi-channel multi-user relaying," in Proc. IEEE Conf. INFOCOM, Mini-Conf., Apr. 2011, pp. 76-80.

[16] W. Wang and R. Wu, "Capacity maximization for OFDM two-hop relay system with separate power constraints," IEEE Trans. Veh. Technol., vol. 58, no. 9, pp. 4943-4954, Nov. 2009.

[17] D. Wang, Z. Li, and X. Wang, "Jointly optimal subcarrier and power allocation for wireless cooperative networks over OFDM fading channels," IEEE Trans. Veh. Technol., vol. 61, no. 1, pp. 249-257, Jan. 2012.

[18] T. T. Kim and M. Skoglund, "Diversity-multiplexing tradeoff in MIMO channels with partial CSIT," IEEE Trans. Inf. Theory, vol. 53, no. 8, pp. 2743-2759, Aug. 2007.

[19] S. Tatikonda and S. Mitter, "The capacity of channels with feedback," IEEE Trans. Inf. Theory, vol. 55, no. 1, pp. 323-349, Jan. 2009.

[20] C. E. Shannon, "Channels with side information at the transmitter," IBM J. Res. Develop., vol. 2, no. 4, pp. 289-293, Oct. 1958.

[21] S. Bhashyam, A. Sabharwal, and B. Aazhang, "Feedback gain in multiple antenna systems," IEEE Trans. Commun., vol. 50, no. 5, pp. 785-798, May 2002.

[22] J. Choi and R. W. Heath, "Interpolation based transmit beamforming for MIMO-OFDM with limited feedback," IEEE Trans. Signal Process., vol. 53, no. 11, pp. 4125-4135, Nov. 2005.

[23] D. J. Love and R. W. Heath, "OFDM power loading using limited feedback," IEEE Trans. Veh. Technol., vol. 54, no. 5, pp. 1773-1780, Sep. 2005.

[24] M. Hajiaghayi, M. Dong, and B. Liang, "Using limited feedback in power allocation design for a two-hop relay OFDM system," in Proc. IEEE ICC, 2009, pp. 1-6.

[25] G. Zhang, W. Zhan, and J. Qin, "Power allocation in decode-and-forward cooperative OFDM systems using perfect and limited feedback," Chin. J. Electron., vol. 19, no. 2, pp. 291-295, Apr. 2010.

[26] W. Yu and J. M. Cioffi, "FDMA capacity of Gaussian multiple-access channels with ISI," IEEE Trans. Commun., vol. 50, no. 1, pp. 102-111, Jan. 2002.

[27] W. Yu and R. Lui, "Dual methods for nonconvex spectrum optimization of multi-carrier systems," IEEE Trans. Commun., vol. 54, no. 7, pp. 13101322, Jul. 2006. 
[28] S. Boyd and L. Vandenberghe, Convex Optimization. Cambridge, U.K.: Cambridge Univ. Press, Aug. 2004.

[29] K. Seong, M. Mohseni, and J. Cioffi, "Optimal resource allocation for OFDMA downlink systems," in Proc. IEEE ISIT, Jul. 2006, pp. 1394-1398.

[30] V. Luc, L. Jerome, O. Onur, and Z. Abdellatif, "Rate-optimized power allocation for DF-relayed OFDM transmission under sum and individual power constraints," EURASIP J. Wireless Commun. Network., vol. 2009, p. 6, Feb. 2009.

[31] J. Louveaux, R. Duran, and L. Vandendorpe, "Efficient algorithm for optimal power allocation in OFDM transmission with relaying," in Proc. IEEE Int. Conf. Acoust., Speech, Signal Process., Mar. 2008, pp. 3257-3260.

[32] A. Gersho and R. M. Gray, Vector Quantization and Signal Compression. Boston, MA: Kluwer, 1992.

[33] J. G. Proakis, Digital Communications, 4th ed. New York: McGrawHill, 2000.

[34] L. M. C. Hoo, B. Halder, J. Tellado, and J. M. Cioffi, "Multiuser transmit optimization for multicarrier broadcast channels: Asymptotic FDMA capacity region and algorithms," IEEE Trans. Commun., vol. 52, no. 6, pp. 922-930, Jun. 2004.

[35] S. Boyd, L. Xiao, and A. Mutapcic, "Subgradient methods," in Lecture Notes of EE392O. Stanford, CA: Stanford Univ. Press, Oct. 2003.

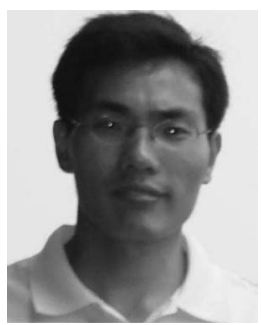

Yong Liu received the B.S. and M.S. degrees in electronic engineering from Taiyuan University of Technology, Taiyuan, China, in 2004 and 2007, respectively. He is currently working toward the Ph.D. degree in electronic engineering with the Department of Electric Engineering, Shanghai Jiao Tong University, Shanghai, China.

He is also with the State Key Laboratory for ISN, Xidian University, Xi' an, China. His current research interests lie in the area of wireless communication, relay cooperation, and orthogonal frequency-

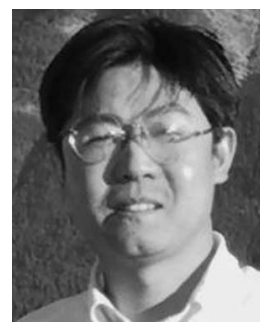

Wen Chen (M'03-SM'11) received B.S. and M.S. degrees from Wuhan University, Wuhan, China, in 1990 and 1993, respectively and the Ph.D. degree from the University of Electro-Communications, Tokyo, Japan, in 1999.

He was a Researcher with the Japan Society for the Promotion of Sciences from 1999 to 2001. In 2001, he joined the University of Alberta, Edmonton, $\mathrm{AB}$, Canada, starting as a Postdoctoral Fellow with the Information Research Laboratory and continuing as a Research Associate with the Department of Electrical and Computer Engineering. Since 2006, he has been a Full Professor with the Department of Electronic Engineering, Shanghai Jiao Tong University, Shanghai, China, where he is also the Director of the Institute for Signal Processing and Systems. He is also with the State Key Laboratory for Integrated Service Networks, Xidian University, Xi' an, China. He has published more than 100 papers in IEEE journals and conferences. His interests cover network coding, cooperative communications, cognitive radio, and mutiple-input-multipleoutput-orthogonal frequency-division multiplexing systems.

Dr. Chen received the Ariyama Memorial Research Prize in 1997 and the PIMS Post-Doctoral Fellowship in 2001. He received the honors of "New Century Excellent Scholar in China" in 2006 and "Pujiang Excellent Scholar in Shanghai" in 2007. He was elected to the Vice General Secretary of Shanghai Institute of Electronics in 2008. He is in the editorial board of the International Journal of Wireless Communications and Networking and serves on the Journal of Communications, Journal of Computers, Journal of Networks, and EURASIP Journal on Wireless Communications and Networking as (lead) Guest Editor. He is the Technical Program Committee Chair for IEEEICCSC2008 and IEEE-ICCT2012 and the General Conference Chair for IEEEICIS2009, IEEE-WCNIS2010, and IEEE-WiMob2011.

division multiplexing systems. 